\title{
Segmentation of Optic Disc and Optic Cup to Calculate CDR using Kekre's LUV Color Space for Detecting Glaucoma
}

\author{
Rajesh B. Awate \\ ME Student \\ Computer Engineering Department TSEC \\ Bandra (w), Mumbai - 50, India
}

\author{
Archana B. Patankar, PhD \\ Associate Professor \\ Computer Engineering Department TSEC \\ Bandra (w) Mumbai - 50, India
}

\begin{abstract}
Glaucoma is the term applied to an eye disease that gradually result in loss of vision by permanently harming the optic nerve, the nerve that transmits visual pictures to the brain. Glaucoma is the second leading cause of blindness worldwide. The retina is the deepest layer in the eye and the retinal nerve filaments transmit the visual sign from the photoreceptors in the eye to the mind through the pack going out of the eye, known as the optic nerve. Glaucoma leads to consistent and expedient harm of the retinal nerve fiber layer and consequently can prompt lasting visual impairment. Thus the finding of glaucoma at a prior stage is imperative for its treatment.
\end{abstract}

Current tests using intraocular pressure (IOP) are not sufficiently delicate for populace based glaucoma screening. Glaucoma can be detected by finding out CDR. CDR Gives Cup to Disc ratio of Optic Cup and Optic Disc. CDR will be finding out by calculating Vertical diameter of Optic cup (OC) and Vertical diameter of Optic disc (OD).

To find out Optic Disc and Optic Cup, Kekre's LUV color space will be applied along with k-means clustering. On the off chance that the CDR proportion surpasses threshold level 0.6 it shows high risk of Glaucoma for the tried patient.

\section{General Terms \\ CDR, VDD, VCD}

\section{Keywords}

Fundus retinal image, glaucoma, k-Means clustering, Kekre's LUV, YCbCr, YUV

\section{INTRODUCTION}

Glaucoma is the term connected to a gathering of eye illnesses that bit by bit result in loss of vision by for all time harming the optic nerve, the nerve that transmits visual pictures to the mind [2]. Glaucoma is the second driving reason for visual impairment around the world. The retina is the deepest layer in the eye and the retinal nerve strands transmit the visual sign from the photoreceptors in the eye to the cerebrum by means of the group going out of the eye known as the optic nerve. Glaucoma prompts ceaseless and rapid harm of the retinal nerve fiber layer and subsequently can prompt lasting visual deficiency. Thus the determination of glaucoma at a prior stage is critical for its treatment [1]. A noteworthy concern with glaucoma recognition is that the malady has no specific arrangement of physical reasons or side effects that specialists can perceive to recognize the sickness in an early stage. The fundamental focus in glaucoma analysis is to recognize changes in the visual working of the eye at ahead of schedule phases of the malady with the goal that vision can be secured and protected through therapeutic treatment [4].

Glaucoma is a bland name for a gathering of illnesses which causes dynamic optic neuropathy and vision misfortune because of degeneration of the optic nerves [3]. It is the second driving reason for visual deficiency, and is anticipated to influence around 80 million individuals by 2020 .

Current tests utilizing intraocular weight (IOP) are not sufficiently touchy for populace based glaucoma screening.

Confinement and segmentation of disc are essential in numerous PC helped determination frameworks, including glaucoma screening [11]. The limitation concentrates on discovering a disc pixel, frequently the middle. This paper concentrates on the segmentation issue and the disc is situated. Its function admirably in the information set for glaucoma screening. The segmentation gauges the disc limit, which is a testing undertaking because of vein impediments, neurotic changes around disc, variable imaging conditions, and so on. A few procedures have been proposed for disc division, which can be generally appointed format based methods, deformable model based techniques and pixel portrayal based frameworks.

An ophthalmologist will inspect Glaucoma by measuring the CDR (Cup to Disk Ratio) which is the vertical's degree stature of the optic cup (OC) and optic disc (OD). Where there are more ganglion dead cells which may reflect in the cup size can be used to gage the probability of Glaucoma. On the off chance that the quality surpasses 0.6 , it shows for a high danger of Glaucoma and can be then tried utilizing different strategies to affirm the illness.

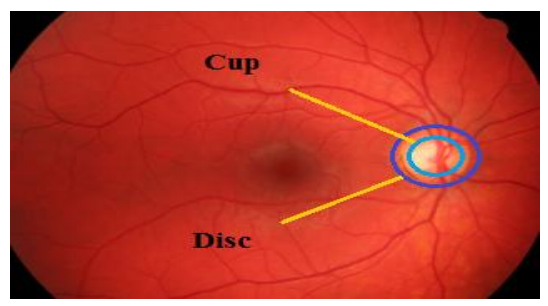

Figure 1: Classification of cup and disc

Prior different systems have been by and by for optic disc discovery [12] however attributable to difficulties because of poor detectable quality of optic cup inside of the optic disc and the apprehensive structural engineering encompassing the optic glass limit tested the last yield. One of the most punctual 
reported techniques was in light of the unfair investigation of shading power [7]. Variation level set in view of pixel force was utilized to universally streamline the got glass form.

According to Yuji Hatanaka's system that investigated taking into account the vein areas that were un-deleted influenced the profile the difference of fleeting side of the optic disc was high in the blue segment picture which influenced its viability [9]. In another methodology by Gopal Datt Joshi, locale based dynamic shape system which dodged force varieties because of vessels. However the glass twisting might not be uniform because of varieties in vessels.

In this study, intention is to locate an advanced answer for optic disc and optic glass discovery. Post evaluating several segmentation methods based on color space.

\section{PROPOSED METHOD}

The proposed framework for the most part comprises of two unique stages. They are Region of Interest (ROI) Extraction, CDR Calculation. In order to extract the optic disc and cup, a region of interest around the optic cup and disc must first be delineated. The disc and cup extraction can be performed in the whole image limiting the ROI (Region of interest) would help to lessen the computational expense and additionally enhance the division exactness. The shape deformation inside of the optic disc (OD) is a critical pointer for the recognition of glaucoma. The disk parameters are estimated using the Disc and Cup boundaries [6].

The optic disc region is as a rule of a brighter pallor or higher shading power than the surrounding retinal area. The ROI is portioned to compute the cup and disc zone, limits furthermore structural and textural elements, to localize the boundary exactly morphological element is connected [10].

\subsection{Image Acquisition}

The main phase of any vision framework is the image acquisition stage. After the image has been gotten, different routines for handling can be connected to the picture to perform a wide range of vision assignments obliged today. Be that as it may, if the image has not been gained agreeably then the expected errands may not be achievable, even with the guide of some type of image upgrade [13].

\subsection{Region of Interest}

Effectively determination of the optic disc region of interest

(ROI) will create a littler starting image which takes much lesser time taken to process contrasted with the whole image.

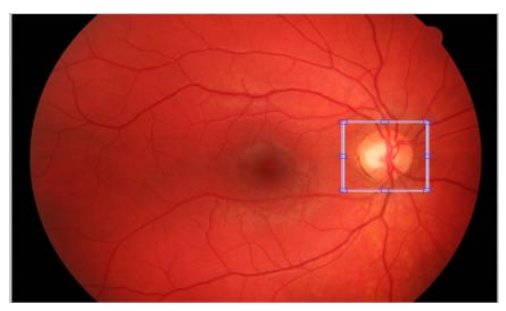

Figure 2: Area of interest

\subsection{Segmentation using K-means clustering}

The K-means is an iterative method that is used to partition the ROI image into K clusters [8]. The ROI covers mainly the whole optic disc, optic cup and a little portion of other regions of the image.

\subsection{Segmentation Using Kekre's Color space}

Segmentation will be done using kekre's LUV color space in color spaces [9], Segmentation will be done based on color of optic disc and optic cup.

\subsection{CDR Calculation and Diagnosis}

After obtaining the disc and cup, different components can be registered. The clinical convention will be follow to compute the CDR [1]. CDR is ratio between the Vertical Cup Diameter and Vertical Disc Diameter.

$$
\mathrm{CDR}=\mathrm{VCD} / \mathrm{VDD}
$$

The processed CDR is utilized for glaucoma screening. At the point when Cup to Disc ratio (CDR) is more noteworthy than a limit, it is glaucomatous generally healthy [5].

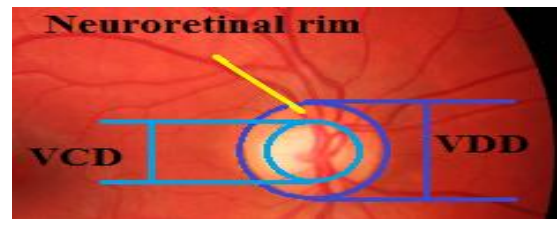

Figure 3: VDD and VCD

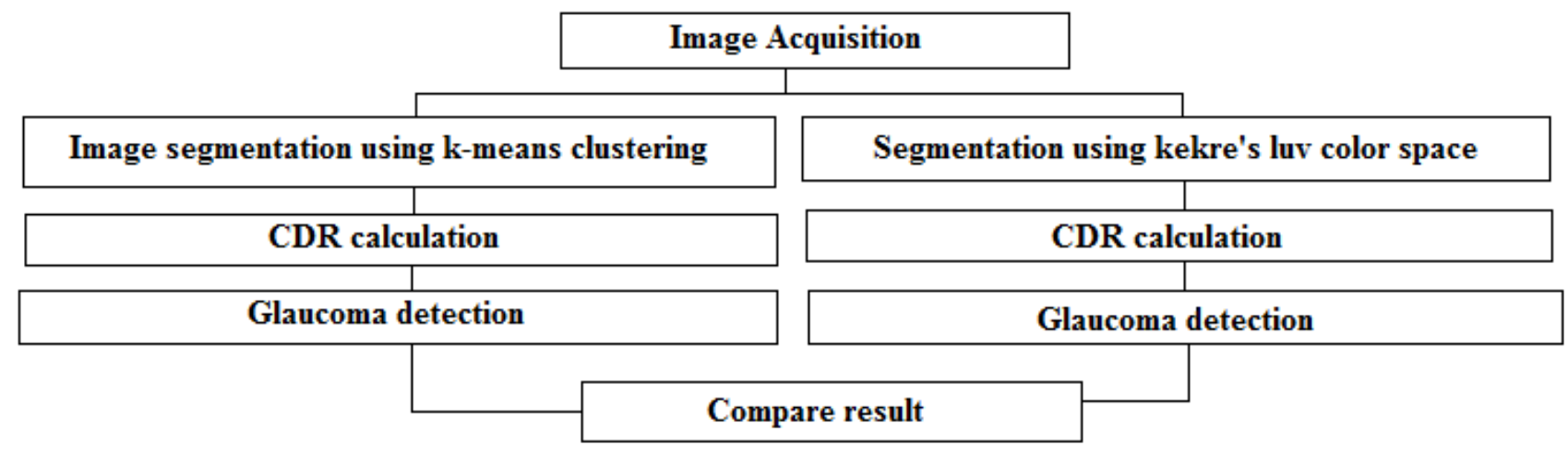

Fig 4: Flowchart of System 


\section{ALGORITHM}

\subsection{K-means algorithm}

Following are the steps in the algorithm

Let $\mathrm{X}=\{\mathrm{X} 1, \mathrm{X} 2, \mathrm{X} 3 \ldots, \mathrm{Xn}\}$ consider as a set of data points and $\mathrm{V}=\{\mathrm{V} 1, \mathrm{~V} 2, \ldots, \mathrm{Vc}\}$ as set of centers.

Randomly select 'C' cluster centers.

Step 1: Figure the separation between every data point and cluster centers.

Step 2: Assign the data point to the cluster center whose distance from the cluster center is minimum of all the clusters.

Step 3: Recalculate the new cluster center using:

$$
\nu_{i}=\left(1 / c_{i}\right) \sum_{j=1}^{c_{i}} x_{i}
$$

Where, ' $c_{\mathrm{i}}$ ' addresses the amount of data points in $\mathrm{i}^{\text {th }}$ cluster.

Step 4: Recalculate the distance between each data point and new obtained cluster centers.

Step 5: If no data point was reassigned then stop, otherwise repeat from step 3)

\subsection{Kekre's color space}

It was evident to stretch out BTC to multi-phantom pictures, for example, shading pictures. Most shading pictures are recorded in RGB space, which is maybe the most understood shading space. Kekre's LUV shading space is unique instance of Kekre change [9]. Where L gives luminance and $\mathrm{U}$ and $\mathrm{V}$ gives chromaticity estimations of shading picture. Positive estimation of $U$ shows conspicuousness of red part in shading picture and negative estimation of $\mathrm{V}$ demonstrates noticeable quality of green segment.

Comparison (1) gives the RGB to LUV change framework which demonstrates the relating $\mathrm{L}, \mathrm{U}$ and $\mathrm{V}$ segments for a picture from the $\mathrm{R}, \mathrm{G}$ and $\mathrm{B}$ segments.

$$
\left[\begin{array}{c}
L \\
U \\
V
\end{array}\right]=\left[\begin{array}{ccc}
1 & 1 & 1 \\
-2 & 1 & 1 \\
0 & -1 & 1
\end{array}\right]\left[\begin{array}{l}
R \\
G \\
B
\end{array}\right]
$$

The opposite transformation, that is from LUV shading space to RGB shading space is given in Equation (2).

$$
\left[\begin{array}{l}
R \\
G \\
B
\end{array}\right]=\left[\begin{array}{ccc}
1 & -2 & 0 \\
1 & 1 & -1 \\
1 & 1 & 1
\end{array}\right]\left[\begin{array}{c}
L / 3 \\
U / 6 \\
V / 2
\end{array}\right]
$$

\subsection{Ycber}

$\mathrm{YCbCr}$ and $\mathrm{Y}^{\prime} \mathrm{CbCr}$ are a down to earth close estimation to shading preparing and perceptual consistency, where the essential hues relating generally to red, green and blue are handled into perceptually significant data. $\mathrm{Y}^{\prime} \mathrm{CbCr}$ is utilized to partitioned out a luma signal $\left(\mathrm{Y}^{\prime}\right)$ that can be put away with high determination or transmitted at high transfer speed, and two Chroma segments (CB and CR) that can be transmission capacity decreased, subsampled, compacted, or generally treated independently for enhanced framework productivity. One pragmatic illustration would be diminishing the data transfer capacity or determination allotted to "shading" contrasted with "highly contrasting", since people are touchier to the highly contrasting data (see picture sample to one side). This is called Chroma subsampling.

Luminance is fundamentally the same to the dark scale form of the first picture. $\mathrm{Cb}$ is solid in the event of parts of the picture containing the sky (blue), both $\mathrm{Cb}$ and $\mathrm{Cr}$ are powerless if there should be an occurrence of shading like green, and $\mathrm{Cr}$ is solid in spots of event of rosy hues [14]. The formulae for changing over from $\mathrm{RGB}$ to $\mathrm{YCbCr}$ are given be.

RGB to YCbCr Conversion

$$
\begin{aligned}
& \mathrm{Y}=(77 / 256) \mathrm{R}+(150 / 256) \mathrm{G}+(29 / 256) \mathrm{B} \\
& \mathrm{Cb}=-(44 / 256) \mathrm{R}-(87 / 256) \mathrm{G}+(131 / 256) \mathrm{B}+128 \\
& \mathrm{Cr}=(131 / 256) \mathrm{R}-(110 / 256) \mathrm{G}-(21 / 256) \mathrm{B}+128
\end{aligned}
$$

YCbCr to RGB Conversion

$\mathrm{B}=1.164(\mathrm{Y}-16)+2.018(\mathrm{U}-128)$

$\mathrm{G}=1.164(\mathrm{Y}-16)-0.813(\mathrm{~V}-128)-0.391(\mathrm{U}-128)$

$\mathrm{R}=1.164(\mathrm{Y}-16)+1.596(\mathrm{~V}-128)$

\subsection{Yuv}

YUV is a picture pressure procedure or some piece of a picture pressure system which overlooks a few subtle elements from the picture, which can't be recognized by human eye. In YUV, Y speaks to luminance or light power of the picture; $\mathrm{U}$ and $\mathrm{V}$ are the shading segments.

The formula is in perspective of the relative responsibilities that red, green, and blue make to the luminance and chrominance variables [15]. There are a few distinct equations being used relying upon the objective screen.

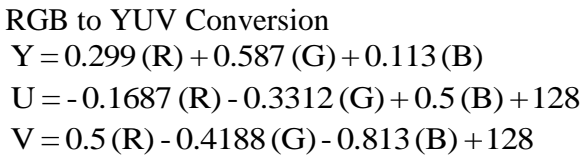

YUV to RGB Conversion

$\mathrm{R}=\mathrm{Y}+0(\mathrm{U})+1.13983(\mathrm{~V})$

$\mathrm{G}=\mathrm{Y}+-0.39465(\mathrm{U})+-0.58060(\mathrm{~V})$

$\mathrm{B}=\mathrm{Y}+-0.03211(\mathrm{U})+0(\mathrm{~V})$

\section{IMPLEMENTATION RESULTS}

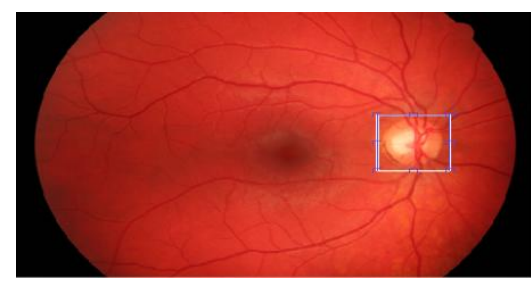

Fig 5: Original image

\subsection{K-Means Results}

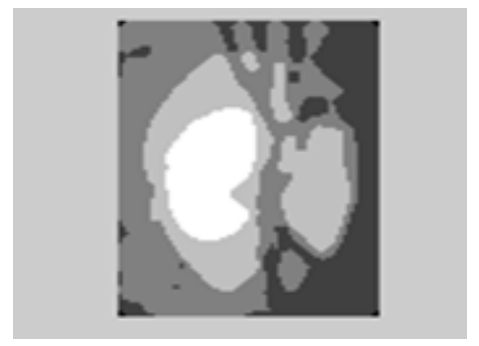

Fig 6: K-means Clustering 


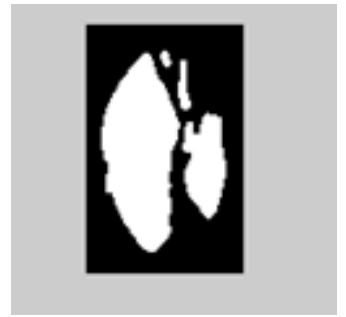

Fig 7: Optic disc

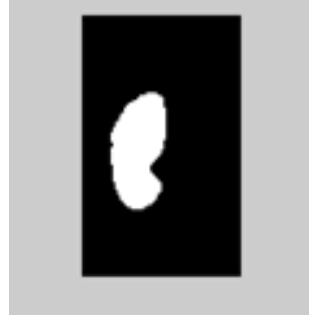

Fig 8: Optic cup

\subsection{Kekre's LUV results}

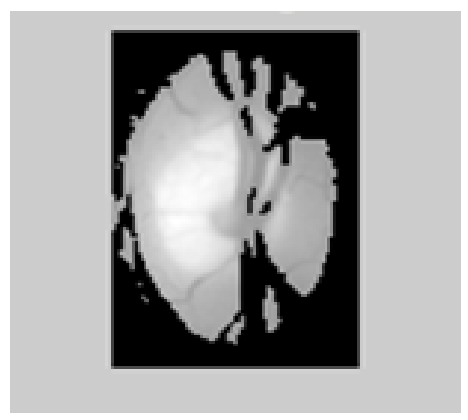

Fig 9: Optic disc

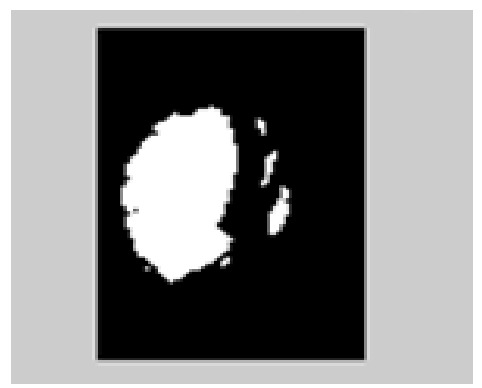

Fig 10: Optic cup

\section{SENSITIVITY AND SPECIFICITY}

Affectability and specificity are factual measures of the execution of a parallel order test, additionally referred to in insights as arrangement capacity:

Affectability (likewise called the genuine constructive rate or the review in a few fields) measures the extent of positives that are effectively distinguished accordingly (e.g., the rate of wiped out individuals who are accurately recognized as having the condition).

Specificity (likewise called the genuine pessimistic rate) measures the extent of negatives that are effectively distinguished as being what is indicated (e.g., the rate of sound individuals who are accurately recognized as not having the condition).

Envision a study assessing another test that screens individuals for a malady. Every individual taking the test either has or does not have the illness. The test result can be sure (foreseeing that the individual has the sickness) or pessimistic (anticipating that the individual does not have the malady). The test results for every subject could possibly coordinate the subject's genuine status. In that setting:
Table1: TPR, TNR, FPR and FNR of proposed and other methods.

\begin{tabular}{|l|l|l|l|l|}
\hline Methods & TPR & TNR & FPR & FNR \\
\hline K-means & 0.81 & 0.72 & 0.28 & 0.19 \\
\hline YUV & 0.62 & 0.65 & 0.35 & 0.38 \\
\hline YCbCr & 0.52 & 0.56 & 0.44 & 0.48 \\
\hline Proposed & 0.9 & 0.8 & 0.2 & 0.1 \\
\hline
\end{tabular}

\section{ROC}

In measurements, a recipient working trademark (ROC), or ROC bend, is a graphical plot that shows the execution of a paired classifier framework as its segregation edge is shifted. The curve is made by plotting the true positive rate against the false positive rate at different limit settings. The genuine positive rate is otherwise called affectability or the affectability record d', known as "d-prime" in sign recognition and biomedical informatics, or review in machine learning. The false-positive rate is otherwise called the drop out and can be computed as (1 - specificity). The ROC bend is consequently the affectability as an element of drop out. As a rule, if the likelihood conveyances for both discovery and false caution are known, the ROC bend can be produced by plotting the combined dissemination capacity (zone under the likelihood dispersion from to ) of the recognition likelihood in the y-pivot versus the aggregate appropriation capacity of the false-alert likelihood in $\mathrm{x}$-hub.

The outcomes show higher AUC in glaucoma screening by the proposed system, contrasted and past techniques.

Table2: AUC of proposed and other methods.

\begin{tabular}{|l|l|}
\hline Methods & AUC \\
\hline K-means & 0.740000000000000 \\
\hline YUV & 0.665000000000000 \\
\hline YCbCr & 0.615000000000000 \\
\hline Proposed & 0.775000000000000 \\
\hline
\end{tabular}

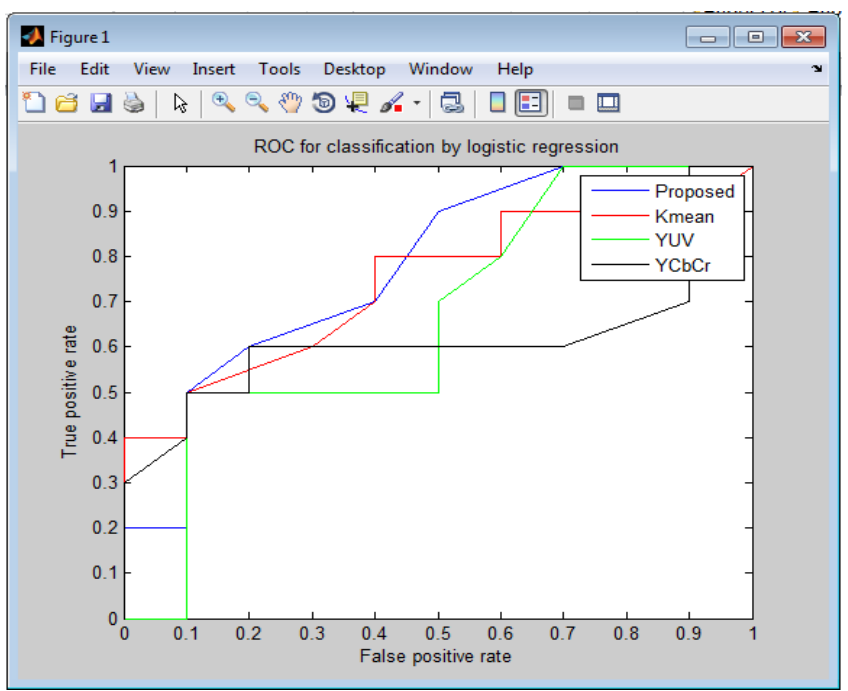

Fig 11: ROC curves of glaucoma screening by previous and proposed $\mathrm{OD}$ and $\mathrm{OC}$ segmentation methods. 


\section{CONCLUSION}

In this paper presented Glaucoma screening using kekre's LUV color space. This paper is exhibited and assessed for Glaucoma detection in patients, including K-Means bunching and kekre's LUV color space of the color fundus camera images to acquire exact limit depiction. Utilizing auxiliary components like CDR (Cup to Disk Ratio), through which it can order as the glaucoma is available or not. This might help in patients worldwide by ensuring further vision decay through convenient restorative mediation. It can increase the number of patients and analyze the performance.

All in all, the cup misshaping is not uniform and the area where the disfigurement happens is likewise utilized by specialists for glaucoma recognition. The k-means procedure took after by the present techniques to acquire the CDR is deficient for this undertaking. This strategy conversely, can be utilized to infer this division insightful data. It should be enhanced to rectify for the cup division by including a part for vessel twist.

\section{ACKNOWLEDGMENTS}

The authors would like to say thanks to Pattern Recognition Laboratory (CS5), the Department of Ophthalmology, Brno University of Technology, Faculty of Electrical Engineering and Department of Biomedical Engineering, Brno (Czech Rep) for giving the database.

\section{REFERENCES}

[1] J Cheng, J Liu, Y Xu, F Yin, D Wong, N Tan, D Tao, $\mathrm{C}$ Cheng, $\mathrm{T}$ Aung, and $\mathrm{T}$ Wong, "Superpixel Classification Based Optic Disc and Optic Cup Segmentation for Glaucoma Screening ," IEEE Trans. Med. Imag., vol. 32, no. 6, pp. 1019 - 1032, Jun. 2013. Ding, W. and Marchionini, G. 1997 A Study on Video Browsing Strategies. Technical Report. University of Maryland at College Park.

[2] A. Aquino, M. Gegundez-Arias, and D. Marin, "Detecting the optic disc boundary in digital fundus images using morphological, edge detection, and feature extraction techniques," IEEE Trans. Med. Imag., vol. 29, no. 11, pp. 1860-1869, Nov. 2010.Tavel, P. 2007 Modeling and Simulation Design. AK Peters Ltd.

[3] H Tjandrasa, A Wijayanti, and N SuciatiOptic , Nerve Head Segmentation Using Hough Transform and Active Contours," TELKOMNIKA ,vol. 10, no. 3, pp. 18601869, July. 2012 .Forman, G. 2003. An extensive

[4] Empirical study of feature selection metrics for text classification. J. Mach. Learn. Res. 3 (Mar. 2003), 12891305.

[5] Z. Zhang, B. H. Lee, J. Liu, D. W. K.Wong, N. M. Tan, J. H. Lim, F. S. Yin, W. M. Huang, and H. Li, "Optic disc region of interest localization in fundus image for glaucoma detection in argali," in Proc. Int. Conf. Ind. Electron. Appl., 2010, pp. 1686-1689.
[6] Y.T. Yu, M.F. Lau, "A comparison of MC/DC, MUMCUT and several other coverage criteria for logical decisions", Journal of Systems and Software, 2005, in press.

[7] G. D. Joshi, J. Sivaswamy, and S. R. Krishnadas, "Optic disk and cup segmentation from monocular color retinal images for glaucoma assessment," IEEE Trans. Med. Imag., vol. 30, no. 6, pp. 1192-1205, Jun. 2011.

[8] Dr. H.B.Kekre, Sudeep D. Thepade, "Image Blending in Vista Creation using Kekre's LUV Color Space", SPITIEEE Colloquium and International Conference, Sardar Patel Institute of Technology, Andheri, Mumbai, 04-05 Feb 2008.A. Aquino, M. Gegundez-Arias, and D. Marin, "Detecting the optic disc boundary in digital fundus images using morphological, edge detection, and feature extraction techniques," IEEE Trans. Med. Imag., vol. 29, no. 11, pp. 1860-1869, Nov. 2010.Tavel, P. 2007 Modeling and Simulation Design. AK Peters Ltd.

[9] Dr. H.B.Kekre, Sudeep D. Thepade, "Boosting Block Truncation Coding using Kekre's LUV Color Space for Image Retrieval", WASET International Journal of Electrical, Computer and System Engineering (IJECSE), Volume 2, Number 3, pp. 172-180, Summer 2008.Z. Zhang, B. H. Lee, J. Liu, D. W. K.Wong, N. M. Tan, J. H. Lim, F. S. Yin,W. M. Huang, and H. Li, "Optic disc region of interest localization in fundus image for glaucoma detection in argali," in Proc. Int. Conf. Ind. Electron. Appl., 2010, pp. 1686-1689.

[10] Dr. H. B. Kekre, Archana Patankar, Hema Ramesh Galiyal, "Segmentation of blast using vector quantization technique", International Journal of Computer Application Security, 72(15), 2013

[11] R. Bock, J. Meier, L. G. Nyl, and G. Michelson, "Glaucoma risk index: Automated glaucoma detection from color fundus images," Med. Image Anal., vol. 14, pp. 471-481, 2010.

[12] R. Bock, J. Meier, G. Michelson, L. G. Nyl, and J. Hornegger, "Classifying glaucoma with image-based features from fundus photographs," Proc. 29th DAGM Conf. Pattern Recognit., pp. 355-364, 2007.

[13] J. Meier, R. Bock,G.Michelson, L. G. Nyl, and J. Hornegger, "Effects of preprocessing eye fundus images on appearance based glaucoma classification," in Proc. 12th Int. Conf. Compute. Anal. Images Patterns, 2007, pp. 165-172.

[14] B. Payette, "Color Space Converter: RGB to YCrCb," Xilinx Application Note, XAPP637, V1.0, September 2002.

[15] Baisa L. Gunjal and Suresh N. Mali -Comparative Performance Analysis of DWT-SVD Based Color Image Watermarking Technique in YUV, RGB and YIQ Color Spaces\| International Journal of Computer Theory and Engineering, Vol. 3, No. 6, December 2011. 